\title{
Granular Avalanches in Fluids
}

\author{
Sylvain Courrech du Pont ${ }^{1}$, Philippe Gondret ${ }^{1}$, Bernard Perrin ${ }^{2}$, and Marc Rabaud ${ }^{1}$ \\ ${ }^{1}$ Laboratoire Fluides, Automatique, Systemes Thermiques (UMR CNRS 7608), \\ Bât. 502, Campus Universitaire, 91405 Orsay cedex, France and \\ ${ }^{2}$ Laboratoire de Physique de la Matiere Condensée (UMR CNRS 8551), \\ 24, rue Lhomond, 75231 Paris cedex, France
}

(Dated: October 25, 2018)

\begin{abstract}
Three regimes of granular avalanches in fluids are put in light depending on the Stokes number St which prescribes the relative importance of grain inertia and fluid viscous effects, and on the grain/fluid density ratio $\mathrm{r}$. In gas $(r \gg 1$ and $\mathrm{St}>1$, e.g., the dry case), the amplitude and time duration of avalanches do not depend on any fluid effect. In liquids $(r \sim 1)$, for decreasing St, the amplitude decreases and the time duration increases, exploring an inertial regime and a viscous regime. These regimes are described by the analysis of the elementary motion of one grain.
\end{abstract}

PACS numbers: 45.70.-n, 45.70.Ht, 46.10.+z,47.55.Kf.

Keywords: Experiment, granular, hydrodynamics.

Granular matter has received much attention from physicists over the past few years [1]. Beyond the fundamental interest in the physics of granular systems which can present some features of either solids, liquids or even gases, the understanding of granular materials is essential in many industrial activities such as pharmacology, chemical engineering, food, agriculture, and so on. Many studies concern the avalanches that may arise on the slope of a granular pile in air. Such granular avalanches occur in various places in Nature, from small scale, as for the building of any sand pile, to large scale, as the event observed after the Mont St-Helen eruption in 1980. Two angles can be defined when building a pile: the maximum angle of stability $\theta_{m}$ at which an avalanche starts and the angle of repose $\theta_{r}$ at which the avalanche stops. Between these two angles is a region of bistability where the grains can either be flowing ("liquid state") or at rest ("solid state"). Many experiments performed with dry grains in a rotating cylinder [2, 3, , 4, 2, 6] showed clearly the existence of these two angles.

To date, no detailed study has focused on the influence of the interstitial fluid for a totally immersed grain assembly. This influence is certainly important in granular avalanche processes, as evidenced by the marked differences observed by geologists between subaqueous and eolian cross strata 77. As a matter of fact, the propagation of subaqueous dunes differs in general from the propagation of eolian dunes even if the slope angles are quite similar: When the transport rate of sand particles is large enough, the flow is continuous in the lee side of the structure in the immersed case, but occurs by successive avalanches in the dry case [7]. This observation prompted geologists to accumulate data on avalanches of sand or beads in rotating drums filled with air or water [8] or even with glycerol mixtures [9], that seemed to show that the amplitude of avalanches decreases and the time duration increases with the fluid viscosity. We have performed an extensive series of experiments to investi- gate the influence of the interstitial fluid on the packing stability and the avalanche dynamics. The analysis of our results obtained with a rotating drum set-up indicate the existence of three regimes: (i) a free-fall regime for which there is no fluid influence and that corresponds to the classical dry regime, and two regimes where the interstitial fluid governs the avalanche dynamics, namely (ii) a viscous regime and (iii) an inertial regime.

Our rotating drum consists of a cylinder of inner diameter $D$ ranging from $8 \mathrm{~cm}$ to $46 \mathrm{~cm}$ and lying on two parallel rotation axes. It is driven by a microstep motor followed by a $1 / 100$ reducer and a rubber transmission so that the cylinder turns at the rotation rate $\Omega$ by step of $10^{-3}$ degree without shocks. The cylinder is half filled with sieved solid spheres of diameter $d$ and density $\rho_{s}$, totally immersed in a fluid of density $\rho_{f}$ and dynamic viscosity $\mu$. The pile is confined between two parallel glass endwalls separated by the gap width $b$. In each experimental configuration, we used a sufficiently large gap width $(b / d>15)$. This study was achieved at low enough rotation rate $\Omega$ to be in the intermittent regime of macroscopic avalanches [4, 6]: The pile slope increases linearly with time at the rate $\Omega$, and then quickly relaxes by a surface avalanche process. A CCD camera aligned along the axis of the cylinder allows for visualization of the rotating pile. Images are taken at regular time intervals and then analyzed to track the pile interface. Except during the avalanches, the interface is found to be linear, with a roughness of the order of one grain size, which justifies the calculation of the averaged slope angle $\theta$ of the pile with the resolution $0.01^{\circ}$.

Two typical recordings for the time evolution of the average slope angle $\theta$ are displayed in Fig. 1 for 230 $\mu \mathrm{m}$ glass beads immersed either in air or water. We focus on two typical parameters of the avalanche dynamics that we found uncorrelated: The avalanche amplitude characterized by the hysteresis angle $\Delta \theta=\theta_{m}-\theta_{r}$, and the avalanche time duration $T$ which is calculated as the 

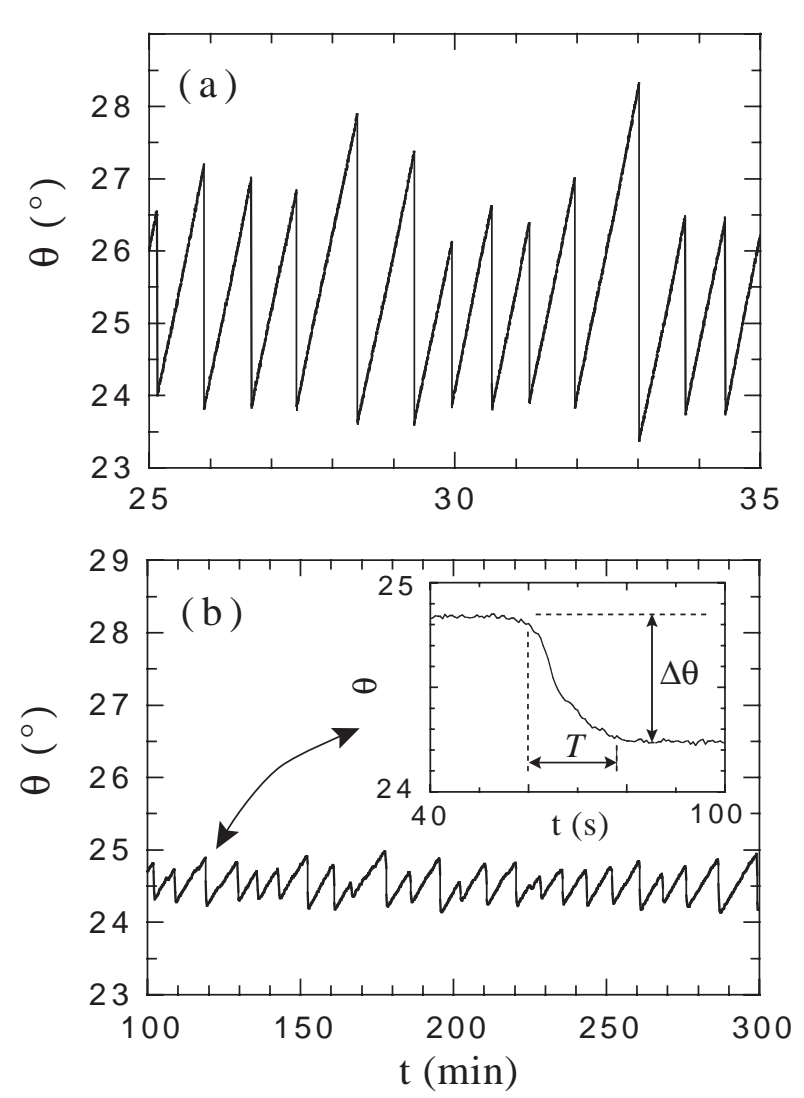

FIG. 1: Time evolution of the average slope angle $\theta$ of a pile of glass beads $(d=230 \pm 30 \mu \mathrm{m})$ immersed in air (a) or water (b) and contained in a rotating drum. The insert is a zoom of an avalanche in the water case.

time interval between $5 \%$ and $95 \%$ of the corresponding avalanche amplitude. The mean values $\overline{\Delta \theta}$ and $\bar{T}$ are then calculated for one experiment over all the successive macroscopic avalanches that affect the entire slope. In the following, we will drop the mean bar notations for simplicity. Despite the dispersion, two different behaviors clearly appear: In the air case (Fig. 1a), the avalanche amplitude $\Delta \theta$ is large (few degrees) and the avalanche duration $T$ is small (typically one second), whereas in the water case (Fig. 1b) $\Delta \theta$ is small (less than one degree) and $T$ is large (typically one minute). One crucial parameter of the phenomenon appears to be the particle diameter $d$ when one looks in Fig. 2 at the evolution of $T$ and $\Delta \theta$ as a function of $d$ when the grains are immersed either in air or in water. In the air case, there is no significant dependency of $T$ and $\Delta \theta$ on $d$. By contrast, in the water case, the avalanche duration which is close to the air case for the larger $d$, increases first sligthly $\left(T \propto d^{1 / 2}\right)$ then drastically $\left(T \propto d^{2}\right)$ from typically 2 to $100 \mathrm{~s}$ when the grain size is decreased from $1 \mathrm{~mm}$ to $0.18 \mathrm{~mm}$. Parallely, the avalanche amplitude $\Delta \theta$ in water corresponds to the air value for the larger $d$ but decreases close to zero when the grain size is decreased to $0.18 \mathrm{~mm}$.

The pertinent dimensionless parameters governing the
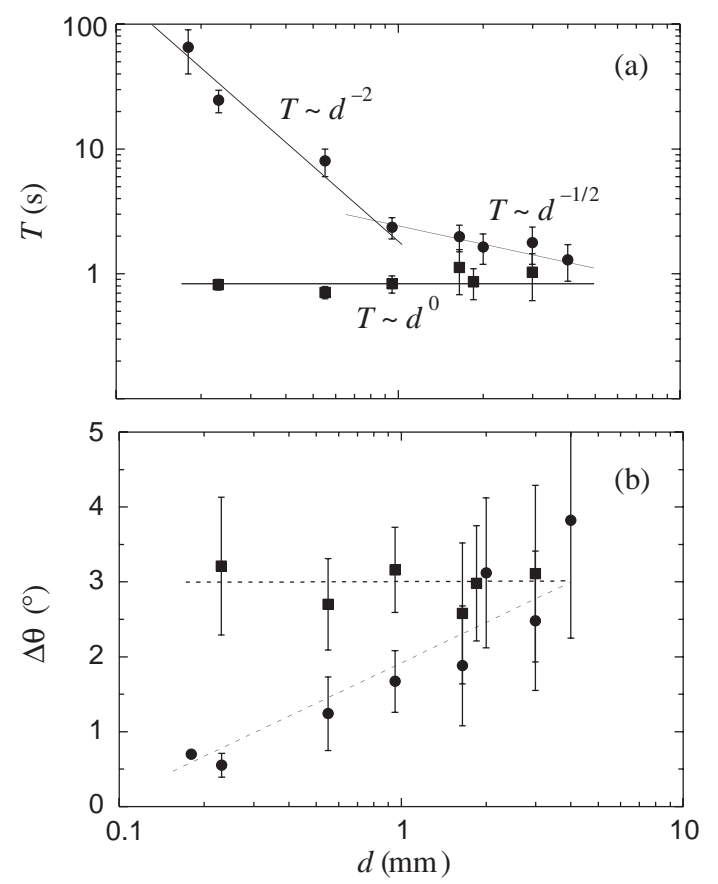

FIG. 2: Time duration $T$ and amplitude $\Delta \theta$ of macroscopic avalanches as a function of the grain diameter $d$ for glass beads immersed in air $(\boldsymbol{\square})$ or water $(\bullet)$ in a rotating cylinder of diameter $D=16 \mathrm{~cm}$. The error bars correspond to the standard deviation.

avalanche dynamics in fluids can be inferred by considering the elementary falling process of one solid grain on its neighbour from below in a fluid and under the action of gravity. Let us write the simplified following equation of motion for the grain of velocity $u$ down the slope between two collisions:

$$
\frac{\pi}{6} \rho_{s} d^{3} \frac{d u}{d t}=\frac{\pi}{6} \Delta \rho g d^{3} \sin \theta-F_{d} .
$$

Starting from zero velocity, the grain increases its grain momentum at the rate $(\pi / 6) \rho_{s} d^{3}(d u / d t)$ under the action of its apparent weigth $(\pi / 6) \Delta \rho g d^{3} \sin \theta$ (where $\Delta \rho$ $=\rho_{s}-\rho_{f}$ ) minus a fluid drag force $F_{d}$. At this basic stage, the solid friction force can be modelled by a dynamical Coulombic term which just reduces the apparent gravity. Two behaviors can be discussed according to the value of the particle Reynolds number: At Re $\ll 1, F_{d}$ is equal to the viscous Stokes force $3 \pi d \eta u$ so the grain will possibly reach its viscous limit velocity $U_{\infty v}=\Delta \rho g d^{2} \sin \theta / 18 \eta$ in the characteristic time $\tau_{c v}=$ $\rho_{s} d^{2} / 18 \eta$, i.e. for a characteristic distance $\delta_{c v}=\tau_{c v} U_{\infty v}$. At Re $\gg 1, F_{d}$ is the inertial fluid force $C_{d}(\pi / 6) d^{2} \rho_{f} u^{2}$, and the inertial characteristic time and distance are $\tau_{c i}$ $=\left(\rho_{s} / \rho_{f}\right)^{1 / 2}\left(2 \rho_{s} d / \Delta \rho g \sin \theta\right)^{1 / 2}$ and $\delta_{c i}=\tau_{c i} U_{\infty i}$ where $U_{\infty i}=\left(2 \Delta \rho g d \sin \theta / \rho_{f}\right)^{1 / 2}$ is the inertial limit velocity (for simplicity we take here the drag coefficient as constant : $C_{d} \simeq 1 / \pi \simeq 0.3$ ). By comparing the two characteristic distances $\delta_{c v}$ and $\delta_{c i}$ with the elementary distance 
beween two successive collisions taken as the grain diameter $d$, we introduce two dimensionless numbers, St and $r$, which govern the grain dynamics in this elementary falling process: $\delta_{c v} / d=2\left(\tau_{c v} / \tau_{f f}\right)^{2}=2 \mathrm{St}^{2}$ and $\delta_{c i} / d$ $=2\left(\tau_{c i} / \tau_{f f}\right)^{2}=2 r^{2}$ where $\tau_{f f}=\left(2 \rho_{s} d / \Delta \rho g \sin \theta\right)^{1 / 2}$ is the typical timescale of free falling of a grain over $d$, St $=(1 / 18 \sqrt{2}) \rho_{s}^{1 / 2}(\Delta \rho g \sin \theta)^{1 / 2} d^{3 / 2} / \eta$ is the Stokes number which prescribes the relative importance of grain inertia and fluid viscous effects of and $r=\left(\rho_{s} / \rho_{f}\right)^{1 / 2}$ is related to the density ratio. Note that the Reynolds number corresponds to the third time ratio, so that $\operatorname{Re}=\tau_{c v} / \tau_{c i}=$ $\mathrm{St} / r$. For St $\gg 1$ and $r \gg 1$, the grain does not reach any limit regime: This is the "free-fall regime". For St $\ll 1$ and $r \gg 1$ the grain reaches its limiting Stokes velocity: This is the "viscous limit regime". For St $\gg 1$ and $r \ll$ 1 , the grain reaches its limiting inertial velocity: this is the "inertial limit regime". For St $\ll 1$ and $r \ll 1$, the grain reaches one limit velocity depending on the $\mathrm{Re}=$ $\mathrm{St} / r$ value: for $\mathrm{Re} \ll 1$ (resp. $\mathrm{Re} \gg 1$ ) the limit regime is the viscous (resp. inertial) one. The exact boundaries between the three domains in the (St, $r$ ) plane of Fig. 3 will be precised further. In this diagram are reported all our data and other data [5, 9], corresponding to different sphere materials (glass and Nylon) in different fluids (air, water, silicone oils or glycerol mixtures of different viscosities). All experimental results correspond to roughly two data lines in this diagram: one data line for the liquid case where $r \sim 1$ and St ranges from 0.2 to 40 and another data line for the air case where $r \sim 40$ and St ranges from 30 to $10^{4}$.

Let us now look if the complex dynamics of macroscopic granular avalanches in fluids can be related to these elementary falling processes. In the two limit regimes, one may reasonably suppose that the time duration $T$ of a macroscopic granular avalanche will scale as $D / d$ elementary fallings each of time duration $d / U_{\infty v}$, so that $T_{v}=D / U_{\infty v}=18 \eta D / \Delta \rho g d^{2} \sin \theta$ in the viscous limit regime and $T_{i}=D / U_{\infty i}=D \rho_{f}^{1 / 2} /(2 \Delta \rho g d \sin \theta)^{1 / 2}$ in the inertial limit regime. By the way, the two scalings $T \propto d^{-2}$ and $T \propto d^{-1 / 2}$ observed in Fig. 2 for small and large $d$ respectively correspond to these two predicted scalings. We have plotted $T / T_{v}$ and $T / T_{i}$ in Fig. $4 \mathrm{a}$ and $4 \mathrm{~b}$ respectively and we observe that all data collapse onto the plateau $T \simeq 4 T_{v}$ for St $\lesssim 5$ (Fig $\left.4 \mathrm{a}\right)$, and the plateau $T \simeq 2 T_{i}$ for $\mathrm{St} \gtrsim 3$ (Fig $\left.4 \mathrm{~b}\right)$. A careful analysis of each data point of Fig 4a,b allow us to identify the critical Reynolds number $\operatorname{Re}_{c} \simeq 2.5$ for the viscous/inertial transition, and thus to draw the corresponding boundary line of slope 1 between the two corresponding domains in the log-log plane (St, $r$ ) of Fig. 3.

In the elementary free-fall regime corresponding to the air case (St $\gg 1$ and $r \gg 1$ ), the same approach would lead to the avalanche time duration $T=(D / d) \tau_{f f}=$ $D\left(2 \rho_{s} / \Delta \rho g d \sin \theta\right)^{1 / 2}$, which is not consistent with the non $d$ dependence observed in Fig. 2. In addition, we

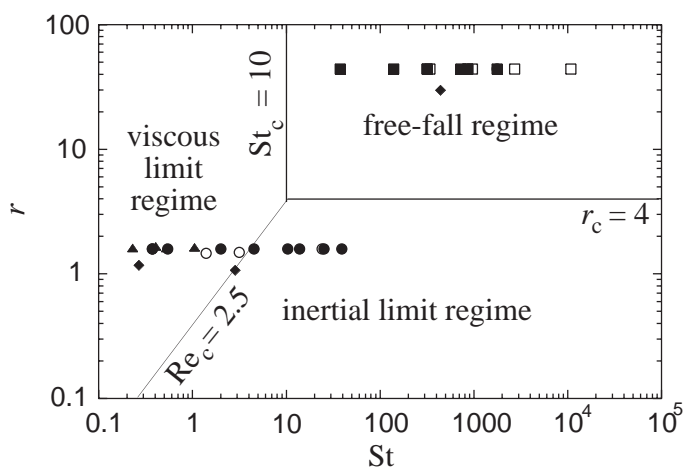

FIG. 3: Diagram of the different regimes for the elementary falling process of one grain in the (St, $r$ ) plane. The three regimes are separated by the three boundaries $\mathrm{St}_{c}=10, \mathrm{Re}_{c}$ $=2.5$ and $r_{c}=4$. Our results (filled symbols) correspond to glass beads in air $(\boldsymbol{\square})$ or water $(\bullet)$, or in silicone oils of different viscosities $(\boldsymbol{\Delta})$, and to Nylon spheres $(\boldsymbol{})$ in air, water and silicone oil. Other results (open symbols) of Evesque [5] $(\square)$ and Allen [10] (o) correspond to glass beads in air and different water-glycerol mixtures.

have also observed that $T$ scales as $D^{1 / 2}$ in the air case rather than as $D$. All these observations lead us to consider a dry avalanche as a global accelerated rush over $D$ of macroscopic timescale $T_{f f}=\left(2 \rho_{s} D / \Delta \rho g \sin \theta\right)^{1 / 2}$ rather than a succession of elementary falling processes. This scaling is indeed observed as all data in air collapse onto the plateau $T \simeq 3 T_{f f}$ for $\mathrm{St} \gtrsim 30$ (Fig. $4 \mathrm{c}$ ). In all three regimes, the plateau values of Fig. $4 \mathrm{a}, \mathrm{b}, \mathrm{c}$ are not far from one, meaning that our crude approach catches the essential of the avalanche dynamics in fluids. Note that for much larger $D$ inaccessible to laboratory experiments, such a free-fall regime will possibly not be observed as the inertial fluid force or a solid friction force will come into play at large grain velocity.

Let us focus now on the avalanche amplitude $\Delta \theta$. When plotting $\Delta \theta$ as a function of the Stokes number St (Fig. 5), we obtain a single master curve. At large St $(\mathrm{St} \gtrsim 20) \Delta \theta$ has the constant plateau value $\Delta \theta \simeq 3^{\circ} \pm 1^{\circ}$ whereas $\Delta \theta$ clearly decreases with $\mathrm{St}$ at small St $(\mathrm{St} \lesssim$ $20)$. We also observe that the decrease of $\Delta \theta$ at small St is essentially due to a decrease of the maximum angle of stability $\theta_{m}$, the angle of repose $\theta_{r}$ being almost unchanged (cf. Fig. 1). This can be explained by considering the collision process beween immersed grains. Indeed, the Stokes number is the only parameter that governs the coefficient of restitution $e$ for the immersed binary collision between solid grains $[10]$. Under the critical value $S t_{c} \simeq 10$, the coefficient of restitution is zero: the collision is totally inelastic as all the kinetic energy of the grain is dissipated by the fluid during the collision process [10]. Above $S t_{c}$, the coefficient of restitution increases quickly with $\mathrm{St}$ and becomes close to its maximal "dry" value $e_{d r y} \simeq 1$ for $\mathrm{St} \gtrsim 100 \sqrt{10}$. It is worthnoting that the curve $e=\mathrm{f}(\mathrm{St})$ is the same whatever 

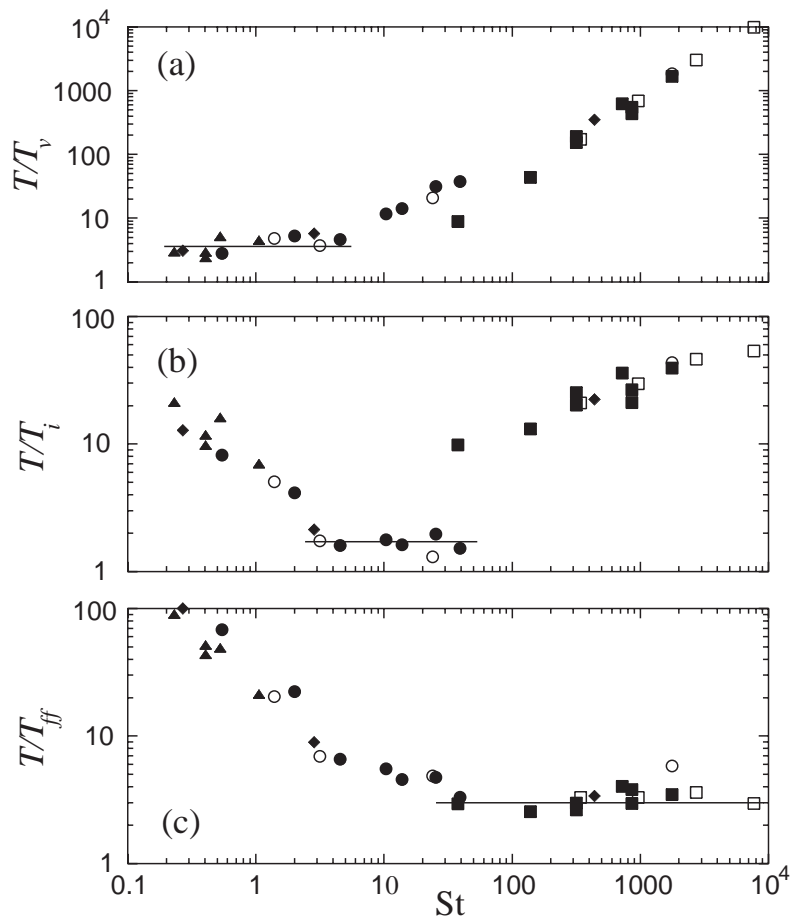

FIG. 4: Time duration $T$ of macroscopic avalanches normalized either by (a) the viscous timescale $T_{v}$ (b) the inertial timescale $T_{i}$ or (c) the free-fall timescale $T_{f f}$ as a function of the Stokes number St for different grains in different fluids and rotating cylinders of different diameter $D$. Same symbols as in Fig. 3.

the density ratio $r$ 10]. Considering again the immersed granular avalanches in fluids, the grain kinetic energy will be totally dissipated by the fluid in the collision process at low St, with an all the more smooth collision when St evolves towards zero. The obtained packing is thus certainly all the more loose, i.e. with a lower packing fraction [11]. As the maximum angle of stability of a granular pile depends largely on the arrangement of the packing, decreasing with the packing fraction [12], this explains the decrease of $\Delta \theta$ with St at low St.

In addition, if one consider the critical Stokes value $\mathrm{St}_{c} \simeq 10$ independent of $r$ as the boundary line between the accelerated regime and viscous limit regime in the (St, $r$ ) plane of Fig. 3, this leads to the critical density ratio $r_{c} \simeq 4$ separating the free-fall regime and the inertial limit regime. As $r$ values larger than $4\left(\rho_{s} / \rho_{f}>\right.$ 16) can hardly be reached experimentally for solid/liquid system, the free-fall regime corresponds only to solid/gas systems like, e.g., the dry granular avalanches.

Finally, for large St (St $\gtrsim 20$ ), all the events correspond to macroscopic avalanches that affect the entire slope and no small event are observed between two successive macroscopic avalanches: The size distribution is a gaussian curve centered on the value $\Delta \theta \simeq 3^{\circ}$. This kind of distribution, classically found for dry granular

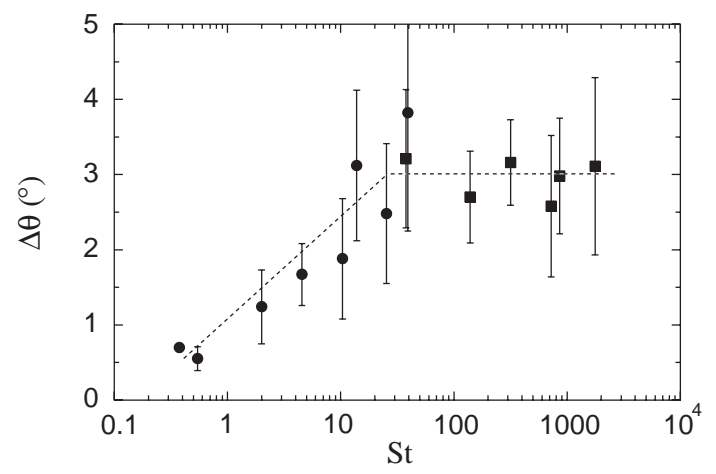

FIG. 5: Amplitude $\Delta \theta$ of macroscopic avalanches of glass beads in different fluids as a function of the Stokes number St. Same symbols as in Fig 3.

avalanches [2, 3, , 1, 5, 6], is incommensurate with the ideas of Self Organized Criticality (SOC) developed by Bak et al. [13] which would predict a power law distribution without any typical scale. The reason may be the dissipation rate of the system [16]: In the cellular automata models, which illustrate nicely the SOC, automata are strongly overdamped whereas dry granular experiments are weakly dissipative. When introducing inertia in cellular automata models, a complex distribution, mixing power law distribution for the small events and gaussian distribution for the large events, is obtained [15]. For decreasing St $(\mathrm{St} \lesssim 20)$, we observe experimentally together with the gaussian distribution of large events the appearance of numerous small events (affecting not all the slope). We expect such a behavior in the viscous regime of low $\mathrm{St}$ as the hysteresis $\Delta \theta$ of the system goes to zero with St, which is a condition for the system to evolve towards criticality. But up to now, we have not enough resolution to characterize the size distribution of these small events, and to conclude if it obeys or not the power law related to SOC. In addition, the regime of intermittent avalanches is hard to obtain when the regime is more and more viscous as the time duration of avalanches diverges.

By conclusion, we have shown the existence of three regimes (free-fall, inertial limit, and viscous limit) for granular avalanches in fluids, controlled by the Stokes number which measures the ratio of particle inertia to viscous fluid effects, and the density ratio. The time duration of the macroscopic avalanches that affect the entire slope have been predicted in all these three regimes. The amplitude of these macroscopic avalanches has been shown to be constant at high St while decreasing with St at low St. Finally, more refined experiments remain to be done to see if the system evolves towards criticality when St tends towards 0, i.e. for highly dissipative systems.

We acknowledge B. Andreotti, S. Douady, D. Lhuillier, O. Pouliquen, E.J. Hinch, and G.M. Homsy for fruitful discussions. 
[1] H.M. Jaeger, S.R. Nagel, and R.P. Behringer, Rev. Mod. Phys. 68, 1259 (1996); P.-G. de Gennes, Rev. Mod. Phys. 71, S374 (1999); J. Duran, Sand, powders, and grains (Springer, New-York, 2000).

[2] P. Evesque and J. Rajchenbach, C. R. Acad. Sci. Paris, srie II 307, 223 (1988).

[3] H.M. Jaeger, C.-H. Liu, and S.R. Nagel, Phys. Rev. Lett. 62, 40 (1989).

[4] J. Rajchenbach, Phys. Rev. Lett. 65, 2221 (1990).

[5] P. Evesque, Phys. Rev. A 43, 2720 (1991).

[6] M. Caponeri et al. in Mobile Particulate Systems, pp. 331-336 (E. Guazzelli and L. Oger eds., Kluwer Academic Publishers, Dordrecht, 1994).
[7] R.E. Hunter, J. Sedimentary. Petrology 55, 886 (1985).

[8] M.A. Carrigy, Sedimentology 14, 147 (1970).

[9] J.R.L. Allen, J. Geology 78, 326 (1970)

[10] P. Gondret, M. Lance, and L. Petit, Phys. Fluids 14, 643 (2002).

[11] G.Y. Onoda and E.G. Liniger, Phys. Rev. Lett. 64, 2727 (1990).

[12] J.R.L. Allen, Geol. Minjbouw 49, 13 (1970).

[13] P. Bak, C. Tang, and K. Wiesenfeld, Phys. Rev. Lett. 59, 381 (1987).

[14] H.M. Jaeger and S.R. Nagel, Science 255, 1523 (1992); D.H. Rothman, J.P. Grotzinger, and P. Flemings, J. Sedim. Res. A64, 59 (1994).

[15] C.P.C. Prado and Z. Olami, Phys. Rev. A 45, 665 (1992). 